\title{
Epidemiological Survey of Frontotemporal Lobar Degeneration in Tottori Prefecture, Japan
}

\author{
Kenji Wada-Isoe ${ }^{a}$ Satoru Ito ${ }^{a}$ Tadashi Adachi $^{\mathrm{a}}$

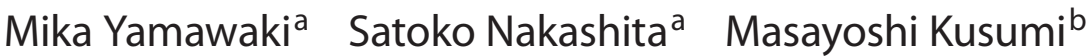 \\ Yu Hiroe $^{c}$ Teruo Takada $^{d}$ Ken Watanabe ${ }^{e}$ Chikanori Hikasa $^{f}$ \\ Kenji Nakashima ${ }^{a}$ \\ a Division of Neurology, Department of Brain and Neurosciences, Faculty of Medicine, \\ Tottori University, ${ }^{\mathrm{b}}$ Department of Neurology, San-in Rosai Hospital, and ' $D e p a r t m e n t ~ o f$ \\ Psychiatry, Yowa Hospital, Yonago, dDepartment of Psychiatry, Saihaku Hospital, Saihaku, \\ and ${ }^{e}$ Department of Psychiatry, Watanabe Hospital, and f Department of Neurology, \\ Welfare Kitazono Watanabe Hospital, Tottori, Japan
}

\section{Key Words}

Prevalence • Frontotemporal dementia $\cdot$ Progressive nonfluent aphasia $\cdot$ Semantic dementia $\cdot$ Tau gene

\begin{abstract}
Background: The prevalence of frontotemporal lobar degeneration (FTLD) in Japan is unknown. An epidemiological survey study of FTLD was undertaken in Tottori Prefecture, a district in the western region of Japan. Methods: Hospitals in Tottori Prefecture were surveyed by a two-step questionnaire in 2010, and the prevalence of FTLD per 100,000 inhabitants was calculated using the actual number of patients and inhabitants in Tottori Prefecture on the prevalence day of October 1, 2010. Results: In this survey, 66 patients were diagnosed with FTLD. The subtypes of FTLD were as follows: 62 cases of frontotemporal dementia (FTD), 3 cases of progressive nonfluent aphasia, and 1 case of semantic dementia. Among the FTD cases, 5 cases were FTD with motor neuron disease and 1 case was FTD with parkinsonism linked to chromosome 17. The prevalence of FTD in the total population of Tottori Prefecture was 11.2 per 100,000 inhabitants. Based on these results, the prevalence of FTLD in Japan in 2008 was estimated to be 9.5 per 100,000 individuals. Conclusions: Our epidemiological survey results suggest that there are at least 12,000 FTLD patients in Japan, indicating that FTLD is not a rare disease.
\end{abstract}




\section{Introduction}

Frontotemporal lobar degeneration (FTLD) is a neurodegenerative disorder predominantly affecting the frontal and temporal lobes. Two major clinical types are recognized in FTLD: behavioral variant frontotemporal dementia (FTD) and progressive aphasia. The latter is divided into progressive nonfluent aphasia (PNFA) and semantic dementia (SD) [1]. Few epidemiological surveys have been conducted concerning FTLD in Japan, and no epidemiological study focusing particularly on FTLD has been reported. In small communitybased studies on the prevalence of dementia in individuals 65 years of age or older, a small percentage of the dementia cases were attributed to FTLD $[2,3]$. In clinic-based survey studies, FTLD was the most third frequent cause of early-onset dementia in patients less than 65 years of age $[4,5]$. The prevalence of FTLD in Japan is unknown. Here, we report a survey study of FTLD in Tottori Prefecture, the least populated district in Japan.

\section{Methods}

We used a questionnaire to perform a retrospective surveillance study of cases of FTLD in Tottori Prefecture. Tottori Prefecture is located in a rural area of western Japan (fig. 1); it had a population of 587,772 (280,602 males and 307,170 females) on the prevalence day of October 1, 2010.

In 2010, we sent inquiries with registration criteria for each category of FTLD to the departments of neurology and psychiatry in the 47 hospitals in Tottori Prefecture where patients with dementia were treated, asking if they had admitted or examined any cases of FTLD during the past year. We then sent a second questionnaire to the departments who responded affirmatively enquiring about the type of FTLD, sex and age of patients, age of onset, symptoms, neuroimaging results, and treatment. If permission was obtained, board-certificated neurologists (K.W.-I., S.I., S.N., and M.Y.) visited the hospitals to examine the patients.

The diagnosis of FTLD was based on the consensus criteria by Neary et al. [1] and the criteria of the International Behavioural Variant FTD Criteria Consortium [6]. Structural neuroimaging [cerebral computed tomography (CT) or magnetic resonance imaging (MRI)] was performed to support the clinical diagnosis. Functional imaging data [cerebral blood flow evaluated by single-photon emission computed tomography (SPECT)] were obtained, if available. Patients were diagnosed as having probable or possible amyotrophic lateral sclerosis using the El Escorial criteria [7, 8].

The prevalence of FTLD per 100,000 inhabitants and 95\% confidence interval (CI) were calculated using the actual number of patients and inhabitants in Tottori Prefecture on the prevalence day, October 1, 2010.

This study was planned and conducted in accordance with the Declaration of Helsinki. The Ethics Committee of the Tottori University Faculty of Medicine approved the study prior to its implementation.

\section{Results}

\section{Survey Results}

Sixty-six patients were diagnosed with FTLD in Tottori Prefecture on the prevalence day (fig. 2). The subtypes of FTLD were as follows: 62 cases of FTD, 3 cases of PNFA, and 1 case of SD. Among the FTD cases, 5 were FTD with motor neuron disease, and 1 was FTD with parkinsonism linked to chromosome 17 (FTDP-17). Overall, the mean age of patients with 
Fig. 1. Location of the surveyed hospitals in Tottori Prefecture. Tottori Prefecture is located in western Japan. The marked hospitals and clinics (circles) were included in our survey.

Fig. 2. Age-specific number of patients with FTLD.
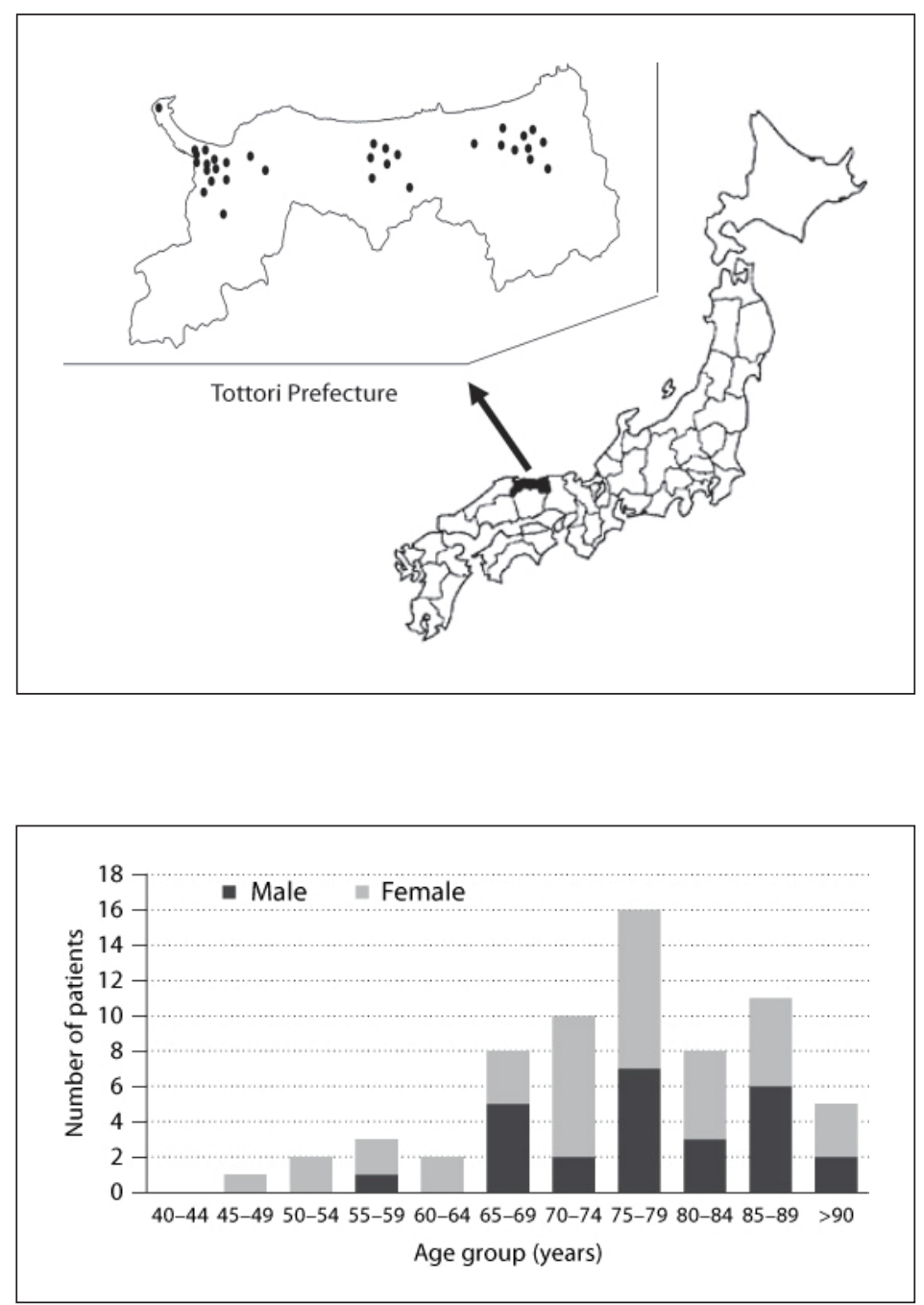

FTD was $76.5 \pm 11.0$ years. The mean age of FTD patients with motor neuron disease was $65.8 \pm 14.3$ years, less than the mean age of FTD patients without motor neuron disease. The mean age of the 3 patients with PNFA was $71.7 \pm 8.1$ years, and the age of the patient with SD was 72 years. Three patients with FTLD had a family history of the disease. Genetic analysis revealed that the patient with FTDP-17 had an intronic mutation IVS10 C $>\mathrm{T}$ in the microtubule-associated protein tau (MAPT) gene.

\section{Prevalence}

Table 1 shows the age-specific prevalence of FTLD per 100,000 inhabitants of Tottori Prefecture in 2010. The survey results indicate that the overall prevalence of FTLD in Tottori Prefecture was at least 11.2 per 100,000 inhabitants. Based on the demographics of Japan in 2008, the data suggest an estimated prevalence of FTLD in Japan of 9.5 per 100,000 inhabitants, or an overall prevalence of at least 12,000 individuals. 
Table 1. Age-specific prevalence estimates of FTLD in Tottori Prefecture

\begin{tabular}{lcc}
\hline & Patients, $\mathrm{n}$ & Prevalence $(95 \% \mathrm{CI})^{1}$ \\
\hline Age group & & \\
$\quad$ 45-54 years & 3 & $4.0(-5.3$ to 8.6$)$ \\
55-64 years & 5 & $5.6(0.7-10.5)$ \\
65-74 years & 18 & $25.8(13.9-37.7)$ \\
$75-84$ years & 24 & $40.5(24.3-56.7)$ \\
$>85$ years & 16 & $64.0(32.6-95.0)$ \\
Population $\geq 45$ years & 66 & $20.7(15.7-26.0)$ \\
Total Population & 66 & $11.2(8.5-14.0)$ \\
\hline
\end{tabular}

${ }^{1}$ Per 100,000 inhabitants.

\section{Discussion}

We conducted an epidemiological survey focusing on the prevalence of FTLD in Tottori Prefecture of Japan. Our previous epidemiological study in Ama-cho [3], a small island town, revealed that only 1 patient with FTLD was diagnosed among 943 subjects with dementia aged 65 years or older, suggesting that more subjects would be needed to examine the true prevalence of FTLD. Therefore, we decided to carry out an epidemiological survey in Tottori Prefecture, which has a total population of 587,772.

The prevalence of FTLD has also been reported in areas outside of Japan. In populationbased studies, the prevalence of FTLD between 45 and 64 years of age has varied from 4.0 per 100,000 individuals in the Zuid-Holland district in the Netherlands to 22 per 100,000 individuals in Brescia, Italy [9-12]. A nationwide hospital-based clinicoepidemiological study in Germany showed a high estimated prevalence of FTLD of 43.1 per 100,000 individuals between 45 and 64 years of age [13]. In contrast, Ikejima et al. [14] reported the prevalence of restricted FTD patient in Ibaraki Prefecture, Japan, to be 2.0 per 100,000 individuals between 45 and 64 years of age. Taken together with our results, the prevalence of FTLD in individuals under 65 years of age in Japan might be less than that in Europe.

Although FTLD is generally considered to be a presenile dementia, a review of demographic characteristics of 353 FTLD patients by Johnson et al. [15] indicated that approximately one quarter of the patients diagnosed as having FTD and semantic dementia and half of PNFA patients had a disease onset after age 65 years. The prevalence of FTLD was 3.8 per 100,000 individuals between 70 and 79 years of age in the Zuid-Holland district in the Netherlands [9]. A nationwide study in Germany estimated the prevalence of FTLD to be 49.3 per 100,000 individuals between 70 and 79 years of age [11]. Gislason et al. [16] reported a much higher prevalence of FTD of 3\% in a cohort of 85 -year-old individuals in Gothenburg, Sweden. In the current study, the prevalence of FTLD was 37.6 per 100,000 inhabitants 65 years of age or older. These epidemiological data indicate that the prevalence of FTLD among elderly subjects might be higher than previously described. One challenging aspect of FTLD is that the clinicopathological features of FTLD in elderly patients may differ from those in patients with presenile-onset FTLD. Baborie et al. [17] proposed that FTLD in elderly patients might exist as a separate entity from presenile-onset FTLD in that the characteristics of clinically frequent memory loss and behavioral changes predominate over language and semantic dysfunction. It was suggested that FTLD in elderly patients is under-recognized, and FTLD should be considered in elderly subjects presenting with an 'atypical Alzheimer's disease' phenotype. 
Although epidemiological studies in Europe have reported that a large percentage of FTLD patients have a family history (29\% in the UK, $43 \%$ in the Netherlands), only $4.5 \%$ of FTLD patients in our current study had a family history. Only 1 patient (1.5\%) of 66 FTLD patients in Tottori Prefecture had a mutation of MAPT, compared with 32 (13.8\%) of 245 FTD patients in the study in the Zuid-Holland district in the Netherlands. These results suggest that genetic factors for the development of FTLD may have a less important role in the Japanese population.

There are several limitations in our estimates of the prevalence of FTLD in Tottori Prefecture of Japan. First, the diagnosis of FTLD was dependent on clinical symptoms only, due to the absence of a definitive biomarker. We could not confirm the diagnosis neuropathologically in any case in this survey. Careful clinical examinations are needed because of the similarities in symptoms between syndromes such as corticobasal degeneration, progressive supranuclear palsy, Alzheimer's disease, vascular dementia, and FTLD. In this study, boardcertificated neurologists and psychiatrists reported the diagnosis of FTLD patients based on their clinical assessment and results of neuroimaging such as cerebral CT, MRI, or cerebral blood flow by SPECT. Further, board-certificated neurologists who have scientific interest in dementia or neurodegenerative disorders visited the clinic or hospital for assessment of the patients when required. For the diagnosis of FTD, we applied the criteria by Neary et al. [5] as well as the criteria of the International Behavioural Variant FTD Criteria Consortium [6]. The former criteria are thought to be relatively insensitive and difficult to apply in the early stage of FTD, whereas the sensitivity of the latter criteria is reported to be better [6].

Further, only patients diagnosed with FTLD who had a medical consultation with the department of neurology or psychiatry were included in the survey. A clinical survey in an academic hospital indicated that the prevalence of PNFA or SD was similar to that of FTD [4]. In the current survey, the proportion of patients with PNFA or SD was much less than that reported in the previous survey, suggesting that the prevalence of PNFA and SD may have been underestimated.

In conclusion, the results of this study suggest that there are as many as 12,000 patients with FTLD in Japan, indicating that FTLD is not a rare disease at all.

\section{Acknowledgment}

We thank the staff at the Department of Neurology at Tottori University for their help in recruiting the patients.

This study was supported in part by the Research Committee of CNS Degenerative Diseases, Ministry of Health, Labor, and Welfare, Japan.

\section{Disclosure Statement}

We certify that there is no conflict of interest. 


\section{References}

-1 Neary D, Snowden JS, Gustafson L, et al: Frontotemporal lobar degeneration: a consensus on clinical diagnostic criteria. Neurology 1998;51:1546-1554.

-2 Ikeda M, Hokoishi K, Maki N, et al: Increased prevalence of vascular dementia in Japan: a community-based epidemiological study. Neurology 2001;57:839-844.

-3 Wada-Isoe K, Uemura Y, Suto Y, et al: Prevalence of dementia in the rural island town of Ama-cho, Japan. Neuroepidemiology 2009;32:101-106.

-4 Yokota O, Sasaki K, Fujisawa Y, et al: Frequency of early and late-onset dementias in a Japanese memory disorders clinic. Eur J Neurol 2005;12:782-790.

-5 Shinagawa S, Ikeda M, Toyota Y, et al: Frequency and clinical characteristics of early-onset dementia in consecutive patients in a memory clinic. Dement Geriatr Cogn Disord 2007;24:42-47.

-6 Rascovsky K, Hodges JR, Knopman D, et al: Sensitivity of revised diagnostic criteria for the behavioural variant of frontotemporal dementia. Brain 2011;134:2456-2477.

$\checkmark 7$ Brooks BR: El Escorial World Federation of Neurology criteria for the diagnosis of amyotrophic lateral sclerosis. Subcommittee on Motor Neuron Diseases/Amyotrophic Lateral Sclerosis of the World Federation of Neurology Research Group on Neuromuscular Diseases and the El Escorial 'Clinical limits of amyotrophic lateral sclerosis’ workshop contributors. J Neurol Sci 1994;124(suppl):96-107.

-8 Brooks BR, Miller RG, Swash M, Munsat TL; World Federation of Neurology Research Group on Motor Neuron Diseases: El Escorial revisited: revised criteria for the diagnosis of amyotrophic lateral sclerosis. Amyotroph Lateral Scler Other Motor Neuron Disord 2000;1:293-299.

-9 Rosso SM, Donker Kaat L, Baks T, et al: Frontotemporal dementia in The Netherlands: patient characteristics and prevalence estimates from a population-based study. Brain 2003;126:2016-2022.

10 Ratnavalli E, Brayne C, Dawson K, Hodges JR: The prevalence of frontotemporal dementia. Neurology 2002;58:1615-1621.

-11 Harvey RJ, Skelton-Robinson M, Rossor MN: The prevalence and causes of dementia in people under the age of 65 years. J Neurol Neurosurg Psychiatry 2003;74:1206-1209.

-12 Borroni B, Alberici A, Grassi M, et al: Is frontotemporal lobar degeneration a rare disorder? Evidence from a preliminary study in Brescia county, Italy. J Alzheimers Dis 2010;19:111-116.

-13 Ibach B, Koch H, Koller M, Wolfersdorf M; Workgroup for Geriatric Psychiatry of the Psychiatric State Hospitals of Germany; Workgroup for Clinical Research of the Psychiatric State Hospitals of Germany: Hospital admission circumstances and prevalence of frontotemporal lobar degeneration: a multicenter psychiatric state hospital study in Germany. Dement Geriatr Cogn Disord 2003;16: 253-264.

- 14 Ikejima C, Yasuno F, Mizukami K, Sasaki M, Tanimukai S, Asada T: Prevalence and causes of earlyonset dementia in Japan: a population-based study. Stroke 2009;40:2709-2714.

$\checkmark 15$ Johnson JK, Diehl J, Mendez MF, et al: Frontotemporal lobar degeneration: demographic characteristics of 353 patients. Arch Neurol 2005;62:925-930.

16 Gislason TB, Sjögren M, Larsson L, Skoog I: The prevalence of frontal variant frontotemporal dementia and the frontal lobe syndrome in a population based sample of 85 year olds. J Neurol Neurosurg Psychiatry 2003;74:867-871.

17 Baborie A, Griffiths TD, Jaros E, et al: Frontotemporal dementia in elderly individuals. Arch Neurol 2012, E-pub ahead of print. 